\title{
OBTENÇÃO E CARACTERIZAÇÃO DE COMPÓSITOS DE POLIETILENO DE ALTA DENSIDADE (PEAD) COM FIBRA DE COCO
}

\section{OBTAINING AND CHARACTERIZATION OF HIGH DENSITY POLYETHYLENE COMPOSITE (HDPE) WITH COCONUT FIBER}

\author{
C. R. de ARAUJO ${ }^{1, *}$ e M. S. SAMPAIO ${ }^{2}$
}

${ }^{1}$ Universidade Federal do Rio de Janeiro, Escola de Química, Departamento de Processos Orgânicos, Rio de Janeiro, Rio de Janeiro, Brasil.

${ }^{2}$ Universidade Federal do Rio de Janeiro, Escola de Química, Rio de Janeiro, Rio de Janeiro, Brasil.

${ }^{*}$ Corresponding author. Universidade Federal do Rio de Janeiro, Escola de Química., Departamento de Processos Orgânicos, Rio de Janeiro, Rio de Janeiro,

Brasil. Telefone: +552139387585

e-mail addressl: carla@eq.ufrj.br(C.R. Araujo).

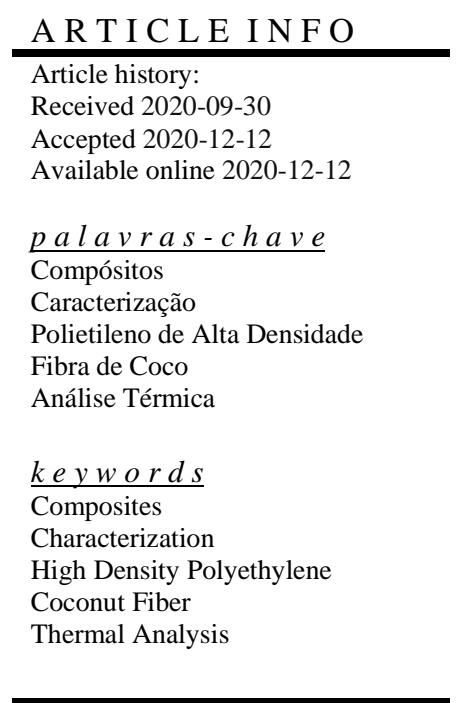

\begin{abstract}
A B S T R A C T
The incorporation of natural fibers is a promising alternative in obtaining composite materials, as its low cost, high availability and unique properties create opportunities for new uses and applications of these fibers. However, the study of the properties of the composites obtained is fundamental in determining the characteristics of the material and its selection process for application in materials, since the composite must meet a series of requirements and properties. With this, the present work had as objective to obtain polyethylene composites with coconut fibers and their characterization through mechanical tests and thermal analysis. The results of the mechanical tests showed that the composites did not show significant changes in tension at maximum strength with the addition of fiber, but showed a decrease in the deformation at break and an increase in the elasticity modulus of the composite with the addition of fiber. The results of the thermal analysis showed that the melting temperature $\left(T_{m}\right)$ of the polymer is $130^{\circ} \mathrm{C}$, the fiber degradation occurs above $200^{\circ} \mathrm{C}$ and the polymer degrades at $485^{\circ} \mathrm{C}$, which allows the processing of the composite without degrading its constituents, although the thermal stability of the composites obtained has decreased with fiber's addition.
\end{abstract}

R E S U M O

A incorporação de fibras naturais se mostra como uma alternativa promissora na obtenção de materiais compósitos, pois seu baixo custo, alta disponibilidade e propriedades únicas criam oportunidades para novos usos e aplicações dessas fibras. No entanto, o estudo das propriedades dos compósitos obtidos é fundamental na determinação das características do material e seu processo de seleção para aplicação em materiais, pois o compósito deve atender a uma série de requisitos e exigências de propriedades. Com isto, o presente trabalho teve como objetivo a obtenção de compósitos de polietileno com fibras de coco e a sua caracterização através de ensaios mecânicos e análise térmica. Os resultados dos ensaios mecânicos mostraram que os compósitos não apresentaram mudanças significativas na tensão na força máxima com a adição de fibra, mas apresentaram uma diminuição da deformação na ruptura e um aumento do módulo de elasticidade do compósito com a adição de fibra. Os resultados da análise térmica mostraram que a temperatura de fusão $\left(T_{m}\right)$ do polímero é de $130^{\circ} \mathrm{C}$, a degradação das fibras ocorre acima de $200^{\circ} \mathrm{C}$ e o polímero se degrada em $485^{\circ} \mathrm{C}$, o que permite o processamento do compósito sem degradar seus constituintes, embora a estabilidade térmica dos compósitos obtidos tenha diminuído com a adição de fibra. 


\section{N O M E N C L A T UR A}

DTA = Análise Térmica Diferencial

DTG $=$ Termogravimetria Derivada

PEAD = Polietileno de Alta Densidade

$\mathrm{TG}=$ Termogravimetria

$\mathrm{T}_{\mathrm{m}}=$ Temperatura de Fusão

\section{INTRODUÇ̃̃̃O}

O desenvolvimento de tecnologias que reduzam o impacto ambiental causado pelo ser humano vem se mostrando como uma tendência mundial devido à crescente preocupação com o meio ambiente. Neste contexto, destaca-se o uso de fibras naturais como reforço na fabricação de materiais compósitos, pois essas fibras são uma fonte renovável promissora e possuem propriedades únicas que as tornam excelentes substitutas às fibras sintéticas (TAJVIDI et al., 2006). Além disso, por serem consideradas como resíduos de outros processos, sua alta disponibilidade e baixo custo atraem especial atenção para o uso dessas fibras na obtenção de compósitos. Dentre as fibras naturais usadas como reforço em materiais compósitos, destacase a fibra de coco, resíduo agroindustrial produzido em larga escala no Brasil (SAMPAIO, 2019).

O polietileno de alta densidade (PEAD) é um dos polímeros mais utilizados na obtenção de compósitos reforçados com fibras, pois é um polímero altamente linear e com estrutura compacta de alta densidade, o que lhe confere uma boa resistência química, boas propriedades mecânicas e menor custo, tornando-o viável para o uso como matriz em compósitos reforçados com fibras vegetais. Desta forma, o desenvolvimento de compósitos de polietileno com fibras de coco se mostra de grande relevância no cenário brasileiro e faz deste material objeto de interesse científico e tecnológico nacional. No entanto, o estudo das propriedades dos compósitos obtidos é essencial na determinação das características do material e no processo de seleção para aplicação em materiais, pois o compósito deve atender a requisitos e exigências de propriedades (SAMPAIO, 2019).

A adesão entre a fibra natural e a matriz polimérica, o volume de fração de fibra, a orientação da fibra e a matriz polimérica utilizada apresentam um papel determinante nas propriedades dos compósitos. A natureza hidrofílica das fibras naturais pode levar a variações na dimensão dos compósitos e também afetar as propriedades mecânicas. As fibras celulósicas, em geral, tendem a agregar, o que dificulta uma distribuição uniforme pela matriz polimérica. Vários estudos foram realizados com o objetivo de aumentar a compatibilidade entre as duas fases - matriz/reforço (ARAÚJO, 2003).

A caracterização de materiais por técnicas como a análise térmica e ensaios mecânicos determina as propriedades e fornece dados de processamento e aplicabilidade dos compósitos obtidos. O objetivo do presente trabalho foi a obtenção de compósitos de polietileno de alta densidade com 5 ,
10 e $20 \%$ de fibra de coco e a sua caracterização por ensaios mecânicos e análise térmica.

\section{MATERIAIS E MÉTODOS}

Materiais:

Polietileno de alta densidade comercial (GM 9450 F);

Compatibilizante Polybond ${ }^{\circledR}$ 3009;

Fibra de coco, resíduo doado pela empresa Coco Verde Reciclado.

Preparo das fibras: As fibras de coco foram secas em estufa por $2 \mathrm{~h}$ na temperatura de $35^{\circ} \mathrm{C}$. Em seguida, as fibras foram moídas e peneiradas, selecionando a fibra que passou pela peneira de 20 mesh.

Preparo dos Compósitos: Os compósitos foram obtidos pela técnica de mistura por fusão, nas proporções de 5, 10 e $20 \%$ de fibra. Foi utilizado um misturador Haake Rheomix 600 com o rotor do tipo Roller. A mistura foi preparada utilizando uma temperatura de $170^{\circ} \mathrm{C}$, velocidade do rotor de $60 \mathrm{rpm}$ e tempo de 10 minutos. Em todos os compósitos foi utilizado $2 \%$ de compatibilizante Polybond® 3009 para melhorar a adesão interfacial entre o polímero e as fibras de coco. A Figura 1 ilustra o PEAD comercial e compósitos obtidos após a mistura.

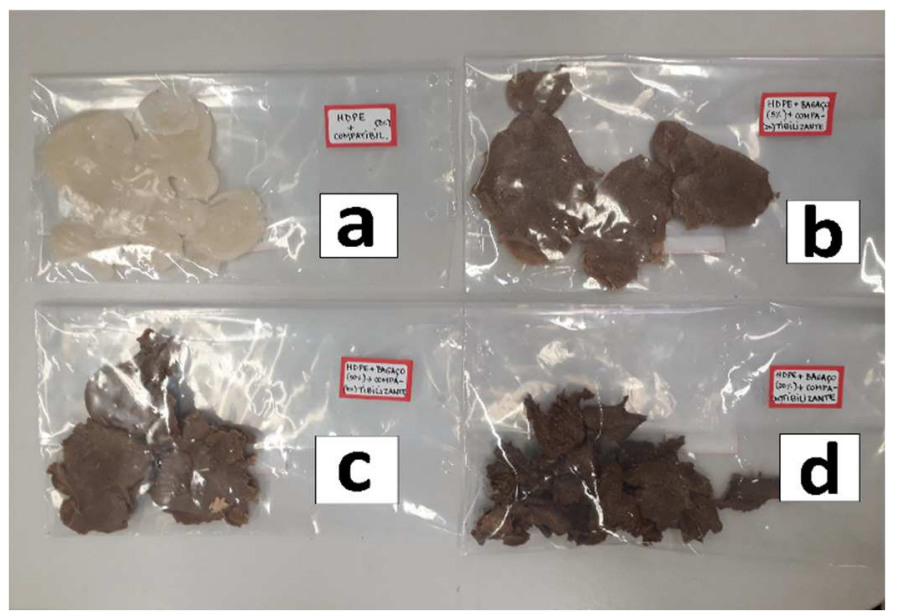

Figura 1 - (a) PEAD comercial e compósitos de PEAD/fibras de coco nas proporções de (b) 5, (c) 10 e (d) $20 \%$ de fibra.

Ensaios Mecânicos: Os ensaios de tração foram realizados em um dinamômetro EMIC, modelo DL3000, segundo a norma ASTM D638. Para a obtenção dos corpos de prova, os compósitos foram prensados em prensa hidráulica de bancada com aquecimento. O tempo de prensagem foi de 4 minutos, na pressão de $10.000 \mathrm{lb} / \mathrm{in}^{2}$ e na temperatura de $170^{\circ} \mathrm{C}$.

Análise Térmica: Foi utilizado um analisador termogravimétrico TA Instruments, modelo STD Q600, com as seguintes condições de análise: faixa de temperatura de 25 à $600^{\circ} \mathrm{C}$, razão de aquecimento de $10^{\circ} \mathrm{C} / \mathrm{min}$; atmosfera inerte (nitrogênio) e massa de aproximadamente $10 \mathrm{mg}$ de amostra. 


\section{RESULTADOS E DISCUSSÃO}

Estudos a respeito das propriedades mecânicas de compósitos poliméricos reforçados com fibras naturais têm sido realizados por muitos autores. A avaliação de propriedades como resistência à tração, deformação na ruptura e módulo de Young dos compósitos depende das propriedades individuais de seus constituintes (ARAÚJO, 2003).

O polietileno de alta densidade com compatibilizante apresentou tensão na força máxima de 21,58 MPa e módulo de Young no valor de $1168 \mathrm{MPa}$. A amostra não apresentou ruptura nas condições de ensaio.

Os compósitos de PEAD com fibra de coco não apresentaram mudanças significativas na tensão na força máxima com a adição de fibra, com valores de 21,47 MPa, 21,86 MPa e 21,32 MPa para os compósitos com 5, 10 e 20\% de fibra de coco, respectivamente, conforme Figura 2. A deformação na ruptura mostrou um decréscimo com a adição de fibra, com valores de $11,71 \%, 9,43 \%$ e $5,65 \%$ para os compósitos com 5 , 10 e $20 \%$ de fibra de coco, respectivamente, conforme Figura 3. $\mathrm{O}$ módulo de Young apresentou crescimento com a adição de fibra, com valores de módulos de 1392 MPa, 1406 MPa e 1634 MPa para os compósitos com 5, 10 e 20\% de fibra de coco, respectivamente, conforme Figura 4. Esses resultados eram esperados, uma vez que o módulo é dependente da concentração da fibra.
A Tabela 1 resume as propriedades mecânicas do PEAD puro e compósitos com 5, 10 e $20 \%$ de fibra de coco.

Tabela 1 - Propriedades mecânicas dos compósitos de PEAD/fibra de coco $(5,10$ e $20 \%)$.

\begin{tabular}{cccc} 
& $\begin{array}{c}\text { Tensão na } \\
\text { Força } \\
\text { Máxima } \\
(\mathrm{MPa})\end{array}$ & $\begin{array}{c}\text { Deformação } \\
\text { na Ruptura } \\
(\%)\end{array}$ & $\begin{array}{c}\text { Módulo } \\
\text { de Young } \\
(\mathrm{MPa})\end{array}$ \\
\hline $\begin{array}{c}\text { PEAD } \\
\text { Puro }\end{array}$ & 21,58 & - & 1168 \\
PEAD & 21,47 & 11,71 & 1392 \\
$5 \%$ coco & & & \\
PEAD & 21,86 & 9,43 & 1406 \\
$10 \%$ coco & & & 1634 \\
PEAD & 21,32 & 5,65 & \\
$20 \%$ coco & & & \\
\hline
\end{tabular}

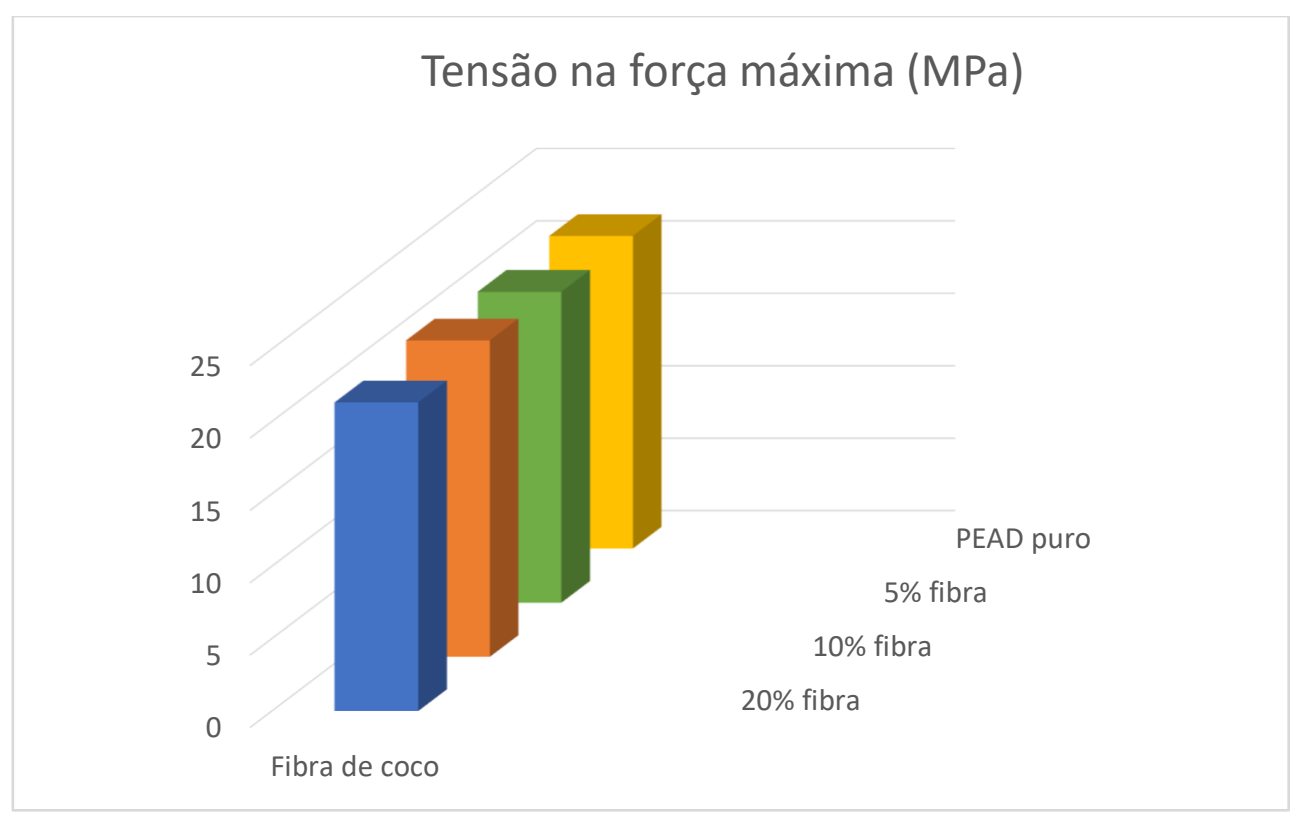

Figura 2 - Valores da tensão na força máxima para o PEAD puro e compósitos com 5,10 e $20 \%$ de fibra de coco e $2 \%$ de compatibilizante. 


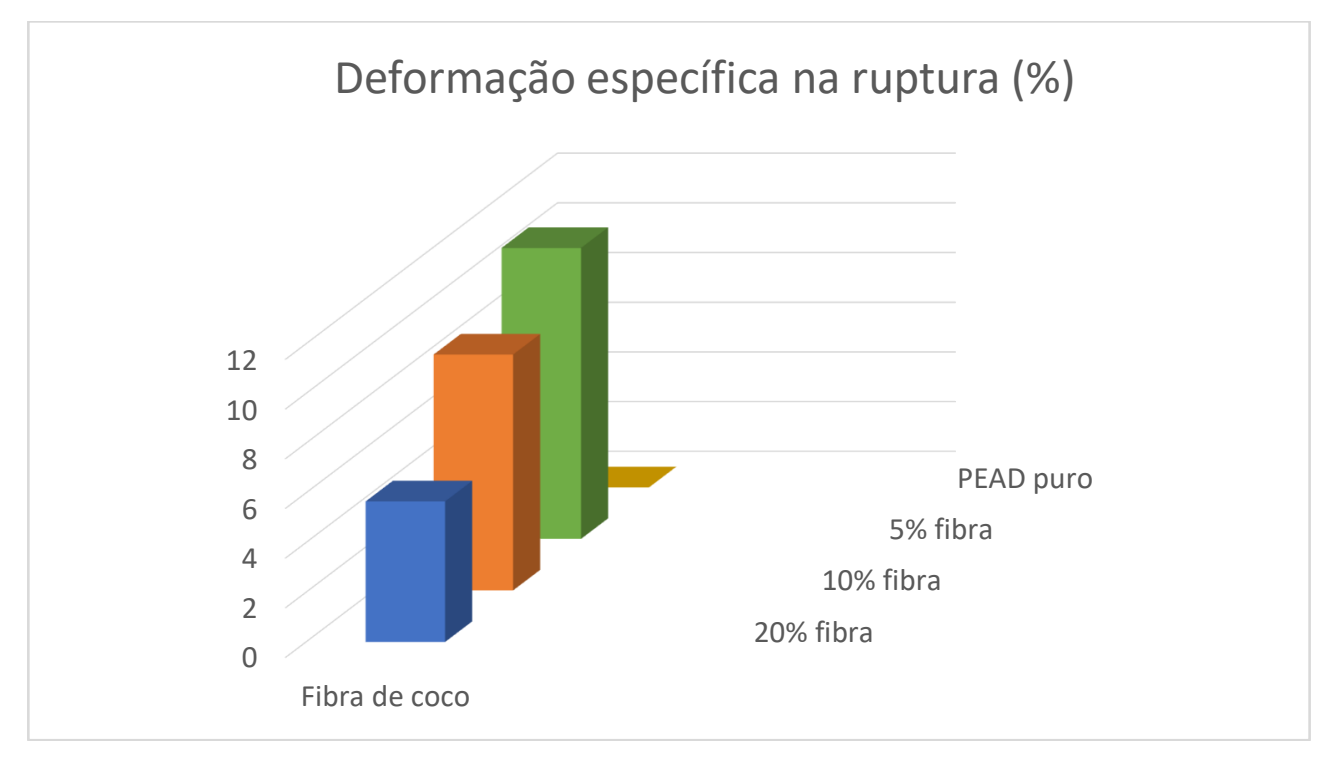

Figura 3 - Valores da deformação específica na ruptura para o PEAD puro e compósitos com 5,10 e $20 \%$ de fibra de coco e $2 \%$ de compatibilizante.

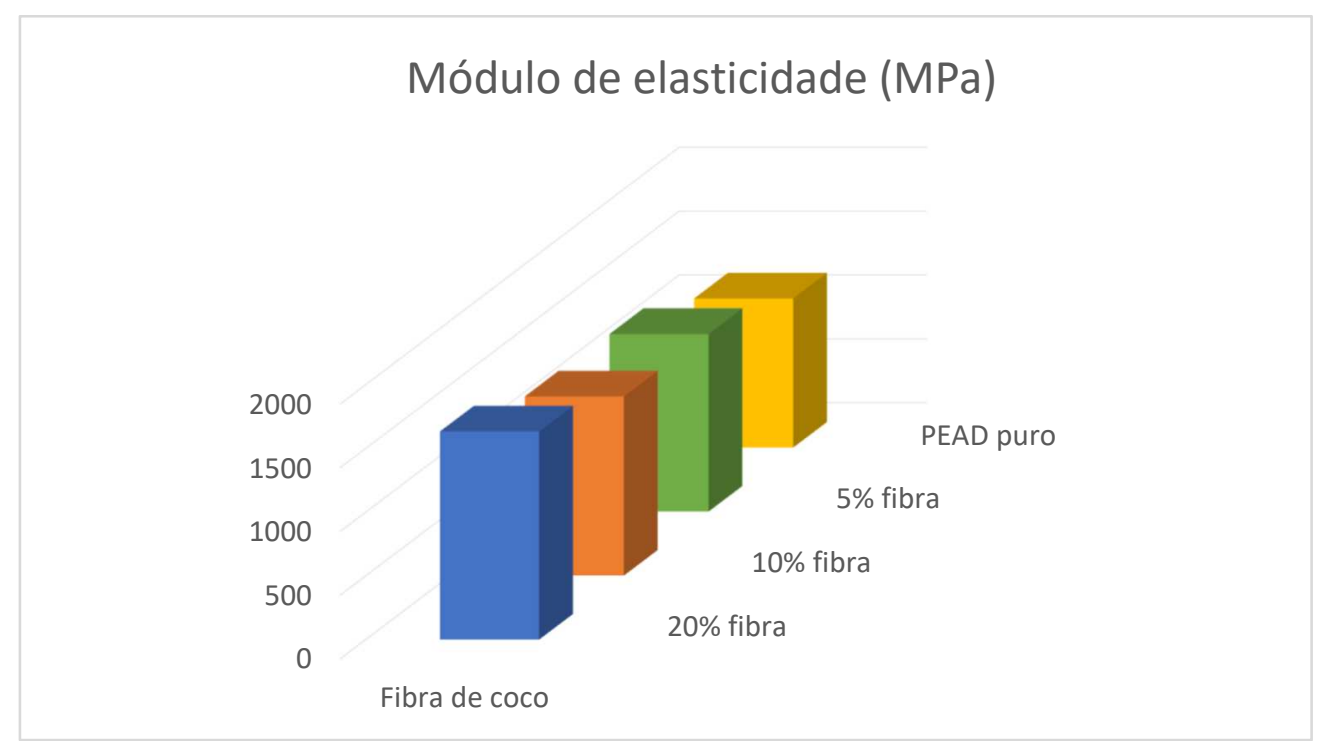

Figura 4 - Valores do módulo de elasticidade para o PEAD puro e compósitos com 5,10 e $20 \%$ de fibra de coco e $2 \%$ de compatibilizante.

O uso das técnicas TG, DTG e DTA serviu para a caracterização térmica das amostras de PEAD com compatibilizante e dos compósitos de PEAD com 5, 10 e $20 \%$ de fibras de coco.

A Figura 5 mostra as curvas TG, DTG e DTA obtidas para o PEAD com $2 \%$ de compatibilizante. A curva TG apresentou um único estágio de decomposição na faixa de 390 a $500^{\circ} \mathrm{C}, \operatorname{com} 100 \%$ de perda de massa, referente à degradação do polímero. Esse resultado foi comprovado pela curva DTG, que apresentou apenas um pico na temperatura de $480^{\circ} \mathrm{C}$. A curva DTA apresentou três eventos endotérmicos: o primeiro a $130^{\circ} \mathrm{C}$, referente à $\mathrm{T}_{\mathrm{m}}$ do polímero; o segundo a $365^{\circ} \mathrm{C}$, referente à presença de aditivos no polímero; e o último a $485^{\circ} \mathrm{C}$, referente à degradação do PEAD. 


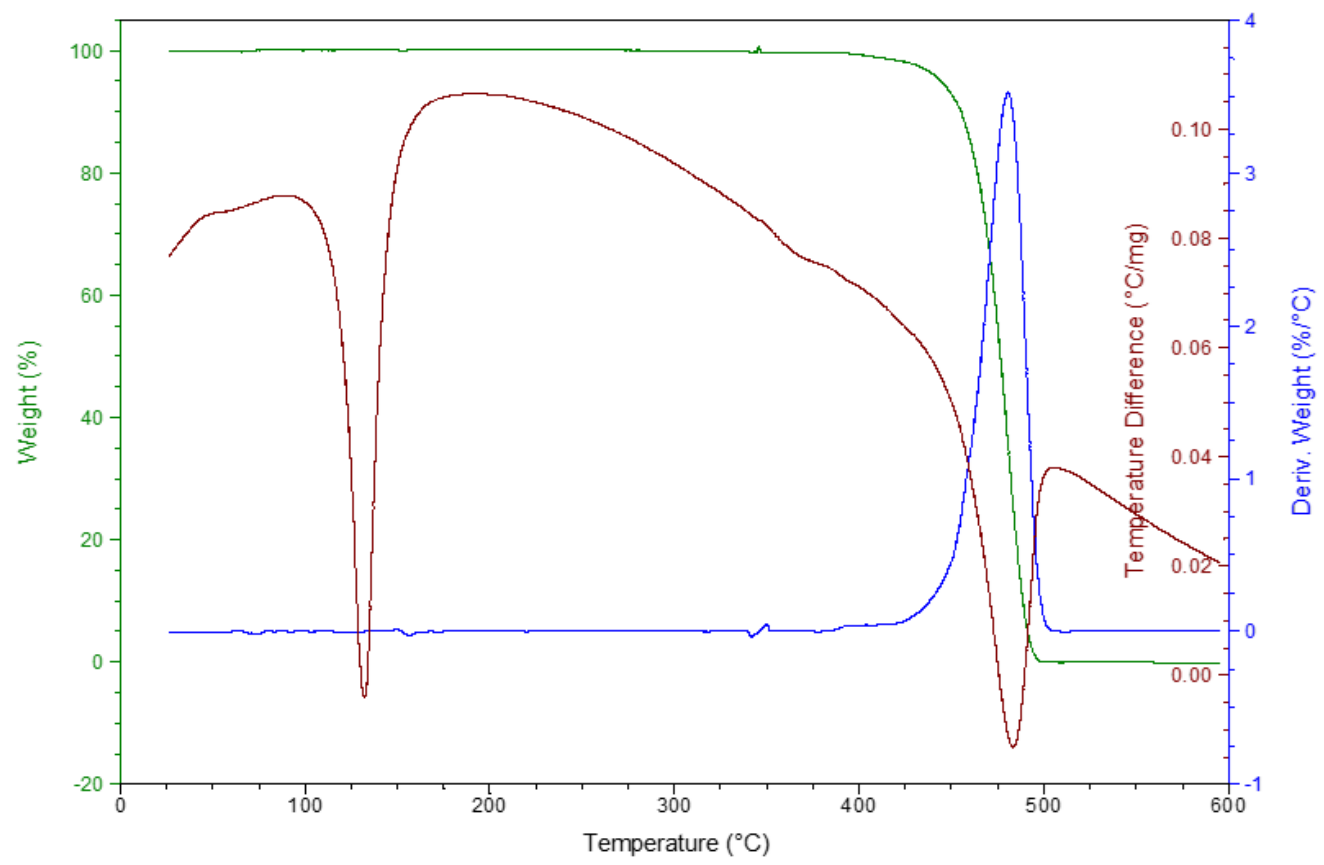

Figura 5 - Curvas TG (verde), DTG (azul) e DTA (vermelha) para o PEAD com 2\% de compatibilizante.

A Figura 6 apresenta a sobreposição das curvas TG para os compósitos de PEAD com 5, 10 e $20 \%$ de fibra de coco e $2 \%$ de compatibilizante. Foi observado que a estabilidade do compósito diminui com a adição de fibra, sendo, portanto, o compósito com $20 \%$ de fibra o menos estável. Isto acontece devido à degradação da fibra, que ocorre antes da degradação do polímero. Quanto mais fibra presente no compósito, menor será a sua temperatura de degradação e maior será a perda de massa no primeiro estágio de decomposição, referente à fibra.

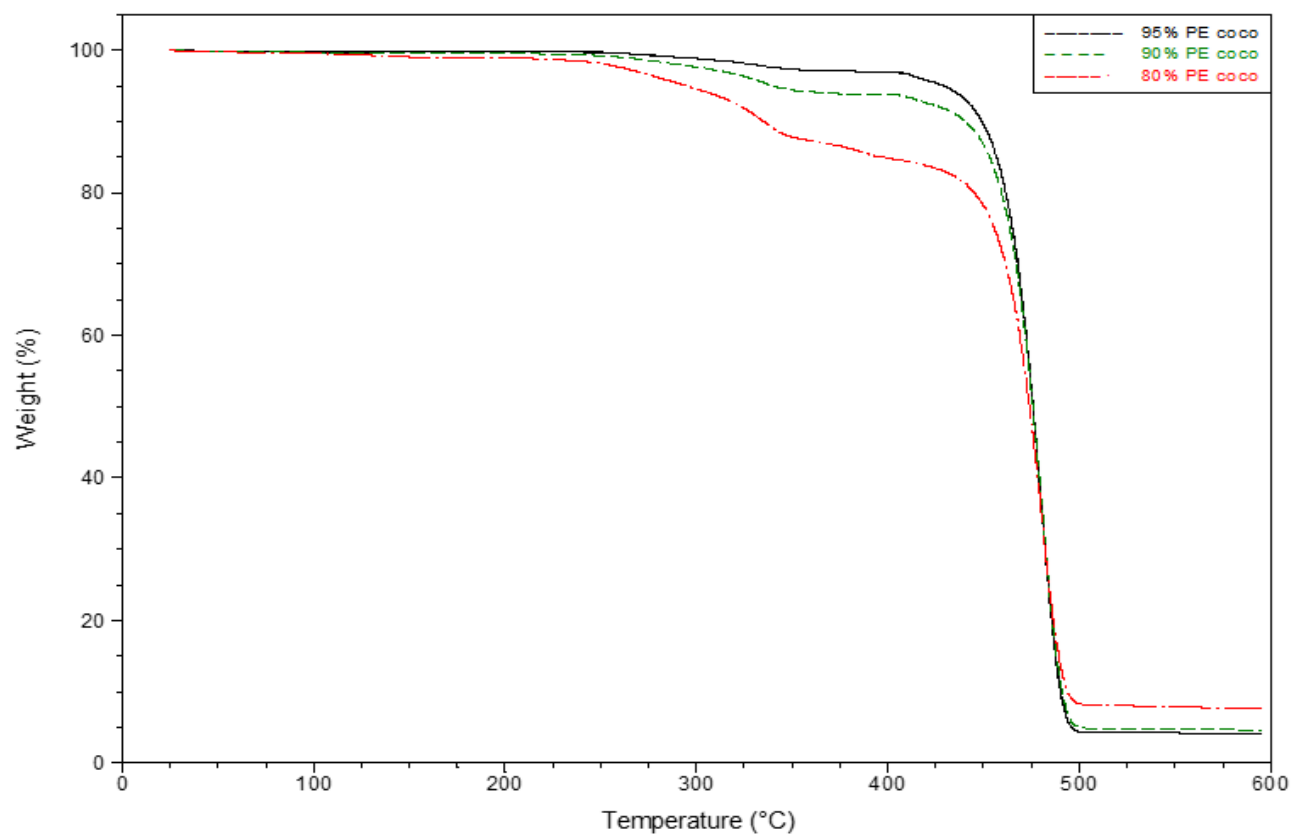

Figura 6 - Sobreposição das curvas TG para os compósitos de PEAD com 5, 10 e $20 \%$ de fibra de coco e $2 \%$ de compatibilizante.

A Figura 7 mostra a sobreposição das curvas DTG para os compósitos de PEAD com 5,10 e $20 \%$ de fibra de coco e $2 \%$ de compatibilizante, que comprovam os estágios observados nas curvas TG. O primeiro pico é referente à degradação da fibra e o segundo pico é referente à degradação do polímero. 


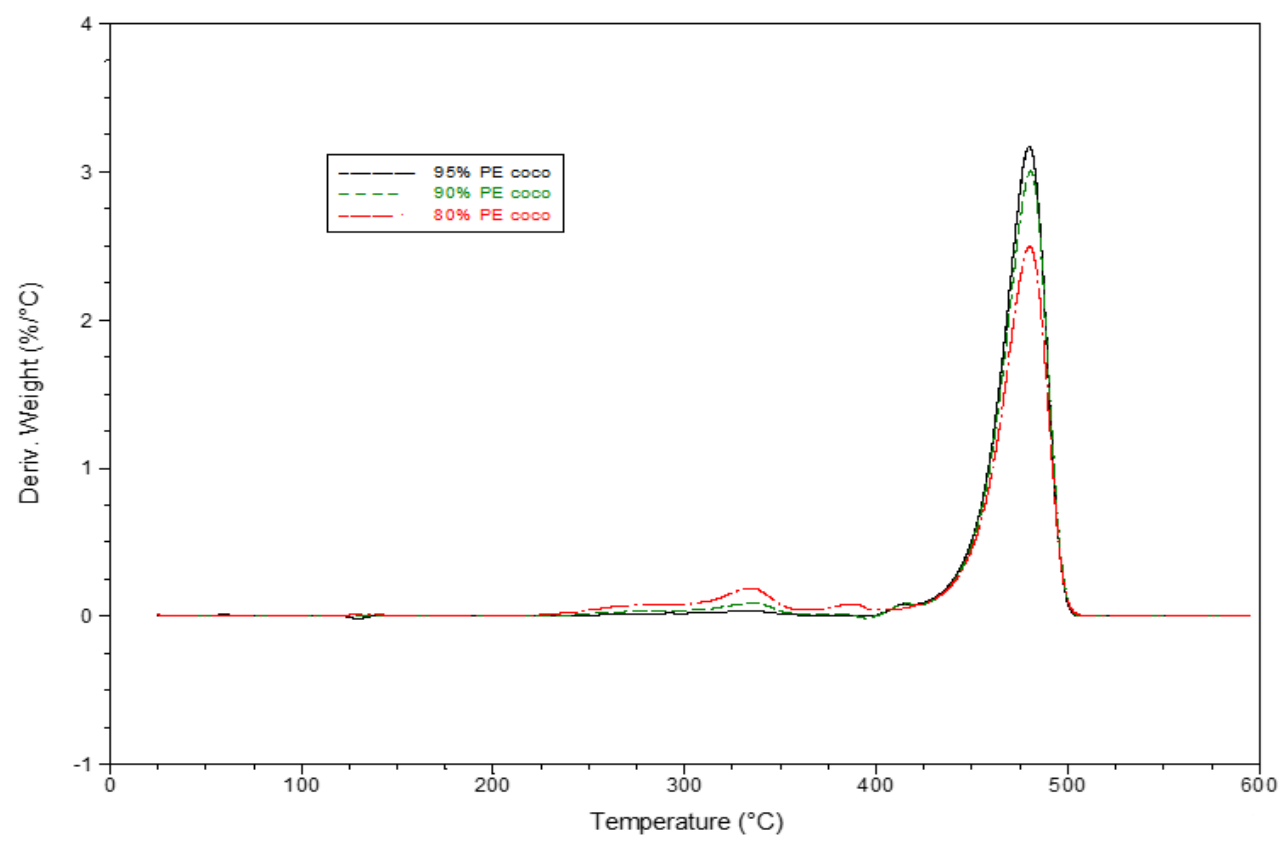

Figura 7 - Sobreposição das curvas DTG para os compósitos de PEAD com 5, 10 e $20 \%$ de fibra de coco e $2 \%$ de compatibilizante.

A Figura 8 ilustra a sobreposição das curvas DTA para os compósitos de PEAD com 5, 10 e 20\% de fibra de coco e $2 \%$ de compatibilizante. Foram observados três eventos endotérmicos, sendo o primeiro evento referente à $\mathrm{T}_{\mathrm{m}}$ do polímero, o segundo evento referente à degradação da fibra e o terceiro evento referente à degradação do polímero.

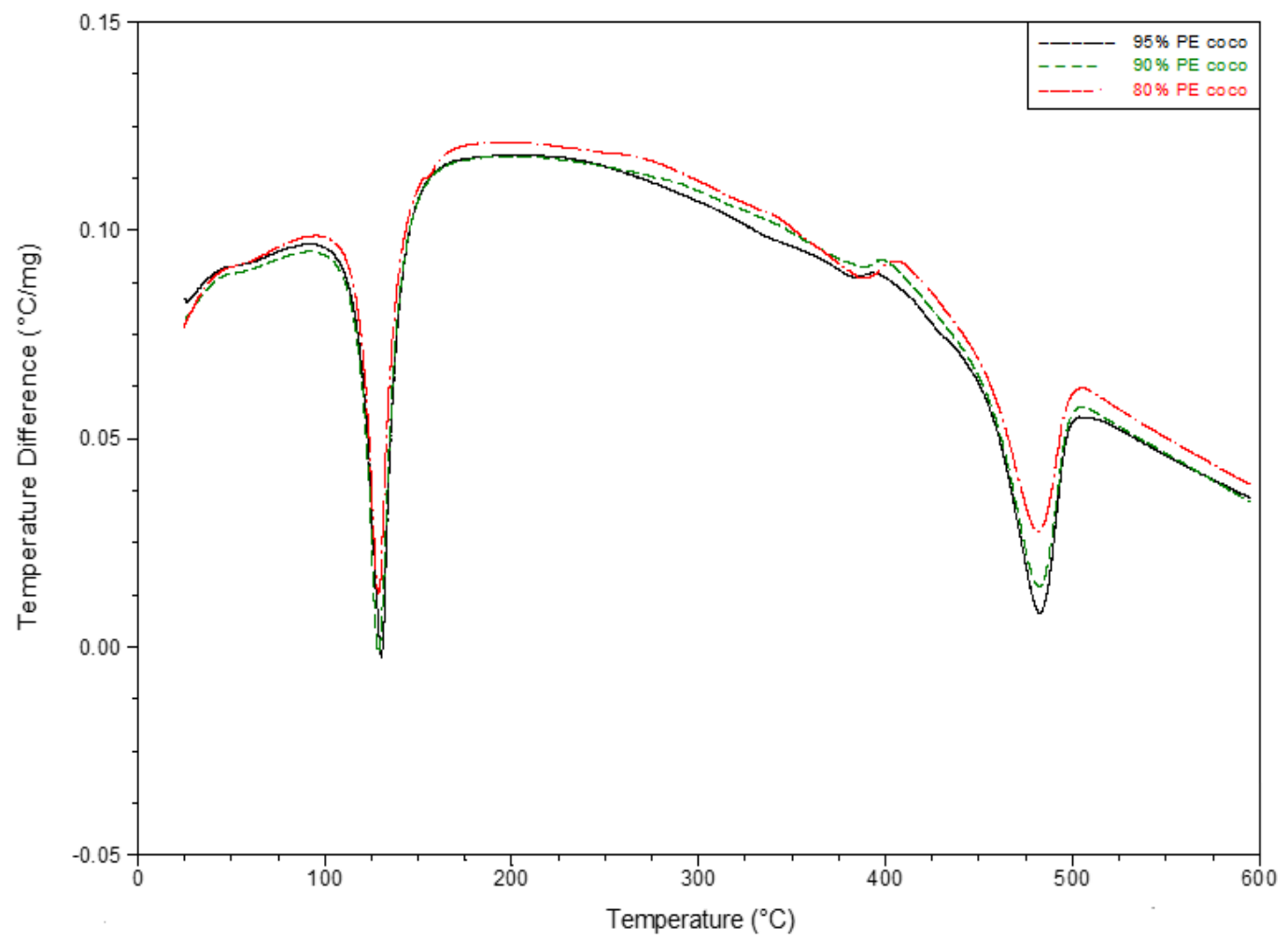

Figura 8 - Sobreposição das curvas DTA para os compósitos de PEAD com 5, 10 e $20 \%$ de fibra de coco e $2 \%$ de compatibilizante. 


\section{CONCLUSÃO}

Os resultados dos ensaios mecânicos mostraram relativa estabilidade da tensão na força máxima dos compósitos. Esse efeito ocorre devido à grande quantidade de lignina na fibra de coco, o que ajuda a promover melhor interação interfacial (BONELLI et al., 2005). Já a deformação na ruptura apresentou decréscimo com a adição de fibra, uma vez que a fibra é mais rígida que o polímero e sua adição provoca uma menor elasticidade do material. Esse resultado foi comprovado também no aumento do módulo de Young com a adição de fibra, pois esta propriedade mecânica mede a rigidez do material e, portanto, deve aumentar com a adição do reforço mais rígido (ARAÚJO, 2003).

A partir dos resultados das curvas TG, DTG e DTA, foi possível notar a diminuição da estabilidade dos compósitos conforme a adição de fibra à matriz polimérica. Isto se deve ao fato de que a degradação da fibra começa antes da degradação do polímero e, portanto, quanto mais fibra no compósito, menor será a temperatura de degradação do mesmo (SAMPAIO, 2019).

Os resultados das técnicas de análise térmica evidenciaram também que, para fins de processamento do compósito, recomenda-se o uso de temperatura por volta de $170^{\circ} \mathrm{C}$, pois o polímero se funde à $130^{\circ} \mathrm{C}$, a degradação das fibras ocorre acima de $200^{\circ} \mathrm{C}$ e a degradação do polímero ocorre em torno dos $485^{\circ} \mathrm{C}$. Com isto, garante-se que o polímero irá fundir e se misturar às fibras sem degradá-las. Já para o uso do compósito em aplicações, recomenda-se uma exposição a temperaturas de no máximo $200^{\circ} \mathrm{C}$, pois acima desta temperatura pode ocorrer a degradação das fibras e perda das propriedades do material.

\section{AGRADECIMENTOS}

Ao Laboratório de Análise Térmica Professor Ivo Giolito - EQ/UFRJ pela realização da Análise Térmica. Ao Instituto de Macromoléculas Professora Eloisa Mano - IMA pela realização dos ensaios mecânicos. Às empresas Fasapel pela doação do PEAD e Coco Verde Reciclado pela doação da fibra de coco. À UFRJ, por incentivar a pesquisa e desenvolvimento no Brasil.

\section{REFERÊNCIAS}

ARAÚJO, C.R. Cinética de decomposição térmica de compósitos poliméricos com fibras de curauá. 2003.71 f. Tese (doutorado em Tecnologia de Processos Químicos e Bioquímicos) - Universidade Federal do Rio de Janeiro, Rio de Janeiro, RJ, 2003.

BONELLI, C. M. C.; ELZUBAIR, A.; SUAREZ, J.C.M.; MANO, E.B. Compósitos de polietileno de alta densidade reciclado com fibra de piaçava. Polímeros: Ciência e Tecnologia, v. 15, p. 256-260, 2005.
SAMPAIO, M.S. Obtenção e caracterização de compósitos de polietileno com fibras naturais. 2019. $62 \mathrm{f}$. Projeto final da graduação (Bacharel em Engenharia Química) Universidade Federal do Rio de Janeiro, Rio de Janeiro, RJ, 2019.

TAJVIDI, M.; FALK, R.H.; HERMANSON, J.C. Effect of natural fibers on thermal and mechanical properties of natural fiber polypropylene composites studied by dynamic mechanical analysis. Journal of Applied Polymer Science, v. 101, p. 4341-4349, 2006. 